\title{
Model organisms-the critical link between gene discovery and therapeutic intervention
}

Jason Berman, MD, FRCPC Departments of Pediatrics, Microbiology \& Immunology and Pathology, Dalhousie University, Halifax, NS

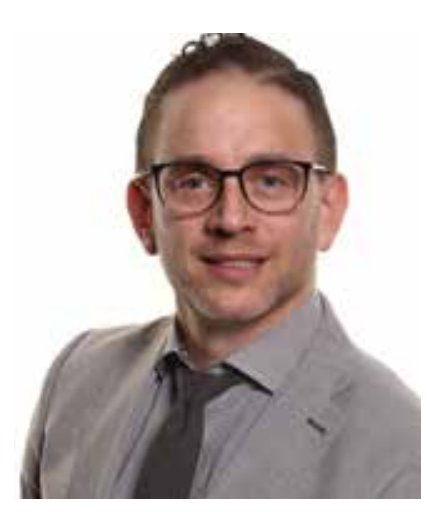

\begin{abstract}
BIO
Jason graduated from the University of Toronto Medical School in 1997 followed by a pediatric residency at the Hospital for Sick Children, a fellowship in pediatric hematology/oncology at Boston Children's Hospital and post-doctoral training at the Dana-Farber Cancer Institute. He is a professor at Dalhousie University in the Departments of Pediatrics, Microbiology \& Immunology and Pathology, was appointed as the director of the Clinician Investigator Program (CIP) and the Graduate Program in Medical Research at Dalhousie (2014), elected president of the Canadian Society for Clinical Investigation (CSCI) (2017) and currently serves as Associate Chair Research in the Department of Pediatrics and Interim VP Research at the IWK, and as an assistant editor of the Journal of Clinical and Investigative Medicine (CIM).
\end{abstract}

\section{Beginnings}

As I reflect on my career to date, it is both curious and understandable how my passion for research and the training of clinician scientists has evolved. Having entered medical school at the University of Toronto after only two years of undergraduate training, I started my residency in Pediatrics at the Hospital for Sick Children with plans to be a clinician and care for children. Even at the outset of my fellowship in Pediatric Hematology/Oncology at Boston Children's Hospital, clinical care seemed to be my calling. I felt rewarded interacting with patients and their families, sharing complex and often devastating diagnostic information and providing the knowledge, guidance and compassion to see them through such a challenging experience. It was not until my second year of fellowship that I entered the laboratory, having very minimal prior research exposure, and this was a game changer. Being somewhat of a late-bloomer with regard to research in my own training is likely the origin of my passion for research training at the post-graduate level, reflected by my involvement in the Royal College Clinician Investigator Program (CIP) at Dalhousie University, where I currently serve as Program Director, and the Canadian Society for Clinical Investigation (CSCl), where I serve as the current President.
Residents and fellows are more differentiated along their clinical specialty tract, such that post-graduate research training has greater potential to inspire a translational research project that may be sustained and evolve into a faculty research program. The best research projects derive from clinical questions-diagnostic limitations, needs for novel therapies, prevention of drug-induced toxicities. This is what fueled my initial foray into research. While I had the opportunity to witness the impressive cure rates provided by multi-agent chemotherapy in diseases such as acute lymphoblastic leukemia (ALL) and Wilms' tumour, I was struck by the poor survival in acute myeloid leukemia (AML), metastatic sarcoma and high risk neuroblastoma. I wanted to better understand these diseases at the molecular level and create more robust preclinical models to identify new treatment strategies.

Since starting my research training, our molecular understanding of childhood and adult cancers has advanced greatly. Much of this gain in knowledge has come from the increasing ability to rapidly and cost-effectively sequence a cancer genome. Recent sequencing efforts, such as the TARGET initiative in the USA (https:// ocg.cancer.gov/programs/target) and the Terry Fox Research Institute sponsored PRecision Oncology For 
Young people (PROFYLE) project in Canada, are revealing detailed exome, genome, methylome, proteome and metabolome profiles of a variety of childhood cancers, providing new information regarding disease biology and potentially targetable malignant driver lesions. However, this deluge of new data, while tremendously exciting, requires adequate bioinformatics to help curate and categorize these findings into clinically actionable pieces of information. Importantly for my work, it also requires functional validation of the significance of a particular genetic alteration, so that it can be appropriately prioritized for therapeutic intervention.

\section{Enter the zebrafish....}

During my fellowship research training, I worked in one of the pioneering laboratories that was just beginning to harness the power of this small vertebrate organism to recapitulate human blood disorders and childhood cancers. Zebrafish have a surprisingly high level of genetic conservation with humans; $>70 \%$ of human proteins have a clear zebrafish orthologue and $>80 \%$ of all human disease-causing genes have a zebrafish orthologue ${ }^{1}$. The functional safeguarding of protein-coding genes has been maintained and their molecular networks are similarly conserved. As a vertebrate, zebrafish provide substantial genetic complexity over simpler model organisms, but are simpler and less costly to maintain than rodents. Cell biology and analogous organs are also present, which are critical for establishing functional significance and evaluating drug metabolism and toxicity. During my training, my lab published the first zebrafish cancer model; a transgenic fish that developed N-myc-driven T-cell ALL². As a new principal investigator, I had the privilege of attending the first Zebrafish Disease Models (ZDM) workshop in Leiden, Netherlands in 2007. As I write this commentary, I am once again in Leiden for the $11^{\text {th }}$ ZDM meeting, which has grown from 70 delegates to a few hundred, highlighting the growth of the field internationally. Zebrafish are now being used to model a wide spectrum of human disorders, from cancer to arthritis to heart disease. Technical advances in the zebrafish model have facilitated the generation of robust representative human disease models. Genetic approaches, in particular the advent of Clustered Regularly Interspaced Short Palindromic Repeats (CRISPR)/
Cas9) technology, has enabled precise genome manipulation to recapitulate germline point mutations, insertions and deletions. We have employed this strategy to model the Li-Fraumeni cancer predisposition syndrome in zebrafish ${ }^{3}$, as well as a number of bone marrow failure disorders and other rare diseases. Transgenic strategies employing tissue specific promoters with inducible temporal regulation can reproduce cancer-causing genetic lesions in somatic cells, and we have exploited transgenesis to generate zebrafish myeloid disease models. Xenotransplantation approaches, which my laboratory has helped pioneer ${ }^{4,5}$, take advantage of the transparency of zebrafish larvae to observe human cancer cell proliferation, migration and interaction with the microenvironment in real time.

The zebrafish, along with other model organisms like flies, worms, yeast and innovative cell-based technologies, in which human-derived cells are placed in a microfluidic apparatus or on a chip, are poised to supplant or at the very least precede typical murine models of human disease. These versatile systems, with their ease of genetic manipulation, provide a critical segue from gene discovery in patients through genotype-phenotype correlations to screening platforms to identify promising new therapies that can be taken back to the clinic. Pipelines that incorporate multiple models, either in sequence (from simple to complex) or in parallel, have the potential to be particularly informative and exclude species or model-specific artifacts. As clinician scientists, we are uniquely positioned to participate in any or all parts of this research continuum across many different clinical specialties. By being astute observers of interesting disease phenotypes, asking important questions regarding etiology and collaborating with clinicians and basic science colleagues in teams fostering complementary expertise, we have tremendous potential to understand the biology of cancer and other genetic disorders and find meaningful new treatment options for patients.

\section{The future}

I have been privileged to work in an academic environment that has supported my ideas and encouraged team science. I have found opportunities to lead, join and advise on scientific pursuits. With a strong supportive base anything is possible, regardless of the size 
of the institution or at what stage one began research training. Be innovative and bold. Seek new partnerships and be open to unexpected offers. Embrace technologic advances (like promising model organisms or genome editing techniques) and by doing so gain recognition as an emerging leader in the field. The post-genomic era is upon us and who better to navigate it than clinician scientists with one foot in the lab and the other in the clinic. $\mathrm{CSCl}$ and the Journal of Clinical and Investigative Medicine will continue to serve as national vehicles to encourage, acknowledge and disseminate the impact of your findings. Go forward from strength to strength.

\section{REFERENCES}

1. Howe et al. Nature.

2013 Apr 25;496(7446):498-503.

2. Langenau et al. Science.

2003 Feb 7;299(5608):887-90.

3. Prykhozhij et al. Nucleic Acids Res.

2018 Jul 24. [Epub ahead of print]

4. Corkery et al. Br J Haematol.

2011 Jun;153(6):786-9.

5. Bentley et al. Haematologica.

2015 Jan;100(1):70-6.

CORRESPONDENCE TO:

E-mail Jason.Berman@iwk.nshealth.ca 